\title{
Evaluación reológica de fluidos de perforación base agua con nanosílice
}

\author{
Felipe Eusebio Gallardo ${ }^{1}$, Eleonora Erdmann ${ }^{1}$, \\ Roxana Abalos ${ }^{1}$
}

\author{
${ }^{1}$ Departamento de Ingeniería en Petróleo - Instituto Tecnológico de Buenos Aires CP: 1106 - Av. Eduardo Madero 399, \\ Buenos Aires, Buenos Aires, Argentina. \\ e-mail: fgallard@itba.edu.ar; erdmann@itba.edu.ar; rabalos@itba.edu.ar
}

\begin{abstract}
RESUMEN
Los fluidos de perforación tienen como función principal remover los recortes de roca generados por el trepano. Son clasificados de acuerdo a sus componentes de base: agua (WBM, water based mud) o aceite (OBM, oil based mud). Entre los aditivos principales encontramos polímeros, densificantes, sales, tensioactivos y lubricantes. La tendencia actual es diseñar WBMs amigables con el ambiente, que puedan competir con los OBMs en términos de baja toxicidad, mínimo daño a la formación productora, eficiencia y bajos costos asociados con la disposición final de los recortes generados y los fluidos. Esto llevó a la incorporación de nuevas formulaciones y materiales tales como los nanométricos.

La creciente demanda hidrocarburífera ha llevado al estudio y producción de reservorios no convencionales de tipo shale en Argentina y el mundo. Este tipo de formaciones sedimentarias de estructura laminar, están conformadas por partículas consolidadas del tamaño de las arcillas, poseen nanoporos y baja permeabilidad. Durante la perforación del shale con WBM se debe garantizar la estabilidad de las paredes del pozo controlando la inhibición de las arcillas presentes y el incremento de la presión poral ocasionado por el ingreso de fluido a la formación. Para este último propósito, en este trabajo, se seleccionó nanosílice de $12 \mathrm{~nm}$, la cual obtura los poros del shale que tienen un tamaño promedio de $30 \mathrm{~nm}$. Se busca de esta forma reducir el ingreso de fluido a la formación, la presión poral, la permeabilidad y minimizar la interacción roca-fluido.
\end{abstract}

Se estudió la reología de los WBM diseñados y envejecidos con distintas proporciones de nanopartículas comparando con el comportamiento reológico de un OBM para determinar la concentración óptima de nanosílice que fue de $0,5 \% \mathrm{p} / \mathrm{p}$. A su vez siguiendo las normas API 13B1-2 y API 13I se realizaron pruebas de control de filtrado e inhibición de los fluidos.

Palabras clave: fluidos de perforación base agua, shale argentino, presión poral.

\section{ABSTRACT}

Generally, drilling fluids may be defined as fluids with a composition that can assist the generation and removal of cuttings from the borehole to the surface. They are classified according to their base as water based mud (WBM), oil based mud (OBM) or gas. Polymers, densifiers, brines, surfactants, among others are the primary components of drilling fluids. Currently the trend is to develop WBMs, which simulate the high performance of OBMs, with benefits such as reduction of environmental impacts and lower costs associated with cuttings and fluids disposal. This challenge introduces high performance materials in the oil industry such as nanomaterials.

The increasing demand of hydrocarbons has led the study and production of unconventional resources in Argentina and worldwide like shale and tight reservoirs. Shales are sedimentary rocks composed of clay size particles and organic matter with laminar structure, low permeability and nanopores. In these types of rocks wellbore stability and shale inhibition are key factors while drilling with WBM, so it is necessary to avoid pore pressure increase and subsequent penetration of drilling fluid into the formation. In this research nanosilica $(12 \mathrm{~nm})$ was used to minimize shale permeability through physically plugging the nanopores of shales which are of $30 \mathrm{~nm}$ as average. This nanomaterial will minimize not only fluid penetration, pore pressure, shale permeability and fluid-rock interaction but will improve wellbore stability.

In this work the rheological behavior of WBM with nanoparticles was analyzed. Fluids were prepared with the same additives and composition as the ones used in argentine fields. Finally, the rheological behavior of 
WBM was compared with the OBM's and the best concentration of nanosilica was determined: $0,5 \% \mathrm{p} / \mathrm{p}$. Also, filtration and inhibition tests were performed in agreement with API 13B1-2 and API 13I.

Keywords: water based mud, argentine shale, poral pressure.

\section{INTRODUCCIÓN}

La creciente demanda de hidrocarburos ha llevado al estudio y producción de reservorios no convencionales del tipo shale en Argentina y en el mundo de forma eficiente y de acuerdo a los requisitos ambientales de cada región. En tal sentido en áreas como la perforación se favorece el diseño de nuevo fluidos de perforación base agua, amigables con el ambiente, que puedan competir y reemplazar los fluidos base aceite (OBM por sus siglas en inglés) en términos de baja toxicidad, mínimo daño a la formación productora, performance y bajos costos asociados con la disposición final de los recortes y fluidos generados. Esto llevó a la incorporación de nuevas tecnologías y materiales a los fluidos como lo son los nanomateriales. [1]

Uno de los aspectos más importantes durante la perforación es garantizar la estabilidad de las paredes del pozo en el tiempo a costos razonables. Para ello se debe controlar el incremento de la presión de hinchamiento que viene dado por el intercambio catiónico con las arcillas del shale, reducir la presión poral asociada a la invasión de fluido de perforación y minimizar la alteración de los enlaces de cementación de la roca relacionado con la difusión iónica en el medio poroso [2]. Por otro lado VAN OORT et al. 1995 [3] y 1996a [4] mostró que el sistema shale-fluido actúan como una membrana semipermeable donde existen dos gradientes uno hidráulico y otro químico gobernados por la leyes de Darcy y Fick respectivamente. Los objetivos antes planteados se pueden alcanzar seleccionando el adecuado tipo de fluido de perforación y sus aditivos.

Los OBMs pueden ser usados prácticamente para cualquier tipo de shale, dado que exhiben mínima interacción química con la roca, al no reaccionar con las arcillas presentes en la formación. Además, la elevada presión capilar que existe entre los nanoporos y el aceite dificulta el ingreso de éste al shale. Las emulsiones inversas, una variedad de OBM, son usadas frecuentemente en las perforaciones realizadas en Argentina a este tipo de roca. Están conformadas por una fase continua de gasoil y una discontinua de salmuera $\left(\mathrm{CaCl}_{2}\right)$ en una proporción cercana a 80:20 en volumen. La salmuera se emplea para minimizar la penetración de agua en el shale ya que posee actividad de agua cercana o menor a la del agua de formación, ocasionando un flujo osmótico desde la formación hacia el pozo. Por todas estas ventajas los OBMs se emplean desde hace años para perforar shales. [5]

Sin embargo, los fluidos de perforación base agua (llamados en ingles WBMs) pueden ser más reactivos dependiendo de las características fisicoquímicas del shale y de los cambios en las condiciones del fluido como temperatura, salinidad, $\mathrm{pH}$ y contaminantes. Por lo tanto, los WBMs deben ser diseñados según la formación a perforar, considerando aspectos técnicos, ambientales y de logística. En estos fluidos se emplean un conjunto de aditivos llamados inhibidores para estabilizar los recortes y reducir la presión de hinchamiento; tales como aminas cuaternarias (por ejemplo politrietanol metilamina y aminas oligoméricas de éter), polímeros encapsulantes (por ejemplo poliacrilamida parcialmente hidrolizada) y sales (por ejemplo $\mathrm{KCl}$ y $\mathrm{NaCl}$ ). Además, se utilizan aditivos para estabilizar las paredes del pozo, llamados aditivos de control de filtrado que controlan la presión poral, como polímeros reductores de filtrado (celulosas, almidones), polímeros para control de la viscosidad (goma xantana, goma guar) y agentes de puenteo (carbonato de calcio, grafito, gilsonita). [6] [7] [8]

La presión poral es un aspecto a considerar dado que el incremento desmedido lleva al desmoronamiento de las paredes del pozo. Para reducir esta presión durante la perforación con WBM se adicionan agentes de puenteo capaces de obturar las gargantas porales de la roca, fisuras y/o fracturas [6] [7]. Los aditivos de control de filtrado que se usan habitualmente, de tamaño entre 0,1 a 100 micrones, no son capaces de formar una torta de filtrado o revoque que evite el ingreso de fluido [9], [10] y demostraron que para bloquear las gargantas porales, el tamaño de las partículas no debe ser superior a la tercera parte del diámetro poral. Dado que el diámetro poral promedio en shales tiene un rango entre 20 a $30 \mathrm{~nm}$ [5] [13] algunos nanomateriales son aptos para bloquear los poros. Esto implica que para perforar shales el fluido de perforación debe tener partículas de tamaño nanométrico, las cuales reducirán la permeabilidad de la roca, la invasión de filtrado y la presión poral. En tal sentido el empleo de nanosílice puede ser una solución económica y eficiente para controlar los problemas de estabilidad de pared del pozo. [14]

JIHUA, et al. (2011) [11] en su trabajo de investigación estudiaron el impacto del uso de seis marcas de nanosílice comerciales, no modificada y con tamaño entre $5 \mathrm{~nm}$ a $22 \mathrm{~nm}$ en fluidos de perforación base agua para reducir la invasión de filtrado. Utilizaron una concentración de $10 \%$ p/p de solución de nanopartículas alcanzando reducciones de permeabilidad entre $45,67 \%$ a $99,3 \%$ en Atoka shales. YOUNG, et al 
(2013) [12] en su investigación determinaron que la concentración de 3\% p/p de nanosílice es la óptima, usando adecuados aditivos de control de filtrado y lubricantes.

Los shales son rocas sedimentarias siliciclásticas de estructura laminar, están conformadas por partículas consolidadas del tamaño de las arcillas y poseen baja permeabilidad y porosidad. [15]. El contenido de minerales arcillosos puede variar significativamente dependiendo del ambiente depositacional, soterramiento e historia diagenética que dieron origen a la roca. En la mayoría de los shales los minerales arcillosos varían entre $15 \%$ a $90 \%$, donde la esmectita puede variar entre $0 \%$ a $75 \%$. Esta arcilla es muy reactiva al agua dado que se hidrata y puede dispersarse en los WBM. El contenido de arcillas define la estructura de la roca y su deformación. Por esta razón es de gran importancia el estudio de la roca y la interacción roca-fluido para el diseño de fluidos de perforación. [16] [17].

El objetivo de esta investigación fue evaluar WBMs con nanomateriales buscando un comportamiento reológico similar a los OBMs utilizados para perforar shales argentinos, sin descuidar propiedades de control de filtrado, inhibición y lubricidad del fluido. La reología es una de las propiedades mas controladas y monitoreadas durante la perforación de un pozo dado que está asociada a la limpieza del mismo, suspensión de recortes y reducción de filtrado. [18] En este estudio se empleó nanosílice inerte de $12 \mathrm{~nm}$ a diferentes concentraciones para luego determinar la concentración óptima de este material. Los ensayos se realizaron de acuerdo a las normas API13B1 y API13B2.

\section{MATERIALES Y MÉTODOS}

Se formularon fluidos de perforación base agua y base aceite con los aditivos utilizados por empresas de servicios que realizan perforaciones en Argentina. Las composiciones de los fluidos estudiados son las que actualmente se emplean en Argentina para perforar formaciones del tipo shale.

Los aditivos de los fluidos de perforación base agua y sus concentraciones se encuentran detalladas en la tabla 1.

Tabla 1: Composición de los fluidos base agua

\begin{tabular}{|c|c|}
\hline \multicolumn{2}{|c|}{ WBM } \\
\hline ADITIVOS & CONCENTRACIÓN \\
\hline Agua & $0,89 \mathrm{~L}$ \\
\hline Bentonita sódica & $10 \mathrm{~g} / \mathrm{L}$ \\
\hline Celulosa polianiónica & $8,0 \mathrm{~g} / \mathrm{L}$ \\
\hline Goma xantana & $1,5 \mathrm{~g} / \mathrm{L}$ \\
\hline Encapsulante & $3,0 \mathrm{~g} / \mathrm{L}$ \\
\hline Amina cuaternaria & $25 \mathrm{ml} / \mathrm{L}$ \\
\hline Lubricante & $10 \mathrm{ml} / \mathrm{L}$ \\
\hline $\mathrm{CaCO}_{3}$ & $60 \mathrm{~g} / \mathrm{L}$ \\
\hline Agente de puenteo & $5,0 \mathrm{~g} / \mathrm{L}$ \\
\hline Baritina & $350 \mathrm{~g} / \mathrm{L}$ \\
\hline Sólidos de formación & $50 \mathrm{~g} / \mathrm{L}$ \\
\hline
\end{tabular}

Esta formulación tiene una densidad de $1.300 \mathrm{~kg} / \mathrm{m}^{3}(10,84 \mathrm{lb} / \mathrm{gal})$ y pH igual a 9 . Cada uno de los aditivos cumple una función específica. La bentonita incrementa levemente la viscosidad del agua y aporta sólidos para el control de filtrado; la celulosa polianiónica o PAC es un polímero de alta calidad, soluble en agua diseñando para controlar el filtrado del lodo y sin modificar sustancialmente la viscosidad; la goma xantana es un polímero natural capaz de modificar la viscosidad del fluido a bajas concentraciones y es tixotrópico; el encapsulante se emplea para estabilizar los recortes generados por el trepano y evitar la dispersión de las arcillas presentes en los mismos; la amina se utiliza para inhibir las arcillas presentes en las paredes del pozo y recortes; el lubricante tiene como función lubricar el trepano y sarta de perforación evitando que los recortes se adhieran; el $\mathrm{CaCO}_{3}$ y agente de puenteo bloquean tanto fisuras y/o fracturas de la roca mejorando el control de filtrado del lodo; la baritina es el principal densificarte del sistema y los sólidos de formación o arcillas simulan el aporte de sólidos luego de la circulación del fluido en el pozo.

La preparación de los fluidos se hizo siguiendo la secuencia establecida por las normas API 13I 
(2004) y usando los equipos descriptos en las normas API 13 B1 (2003). Primero se hidrata la bentonita en agua durante $16 \mathrm{hrs}$, luego se adicionan los polímeros y se agitan durante veinte minutos en mezcladoras a 10000 rpm, a continuación se agrega el lubricante, seguidamente se agregan los agentes de puenteo y densificante, finalmente se adicionan los sólidos de formación; utilizando un tiempo de agitación para cada una de estas etapas de diez minutos.

La nanosílce utilizada es Sigma-Aldrich p.a. (polvo), con un tamaño de $12 \mathrm{~nm}$, inerte, de $201 \mathrm{~m}^{2} / \mathrm{g}$ área superficial y $\mathrm{pH}$ de 4,1. Para su manipulación fue parcialmente hidratada con agua bi-destilada en una relación 8:1. En el caso de los WBMs con nanopartículas, ésta fue agregada a la solución bentonítica y se agitó a altas velocidad de corte durante diez minutos, a continuación, se agregaron el resto de los aditivos.

Los aditivos de los fluidos de perforación base aceite y sus concentraciones se encuentran detallados en la tabla 2.

Tabla 2: Composición de los fluidos base aceite.

\begin{tabular}{|c|c|}
\hline \multicolumn{2}{|c|}{ ОВМ } \\
\hline ADITIVOS & CONCENTRACIÓN \\
\hline Diesel & $0,70 \mathrm{~L}$ \\
\hline Arcilla organofilica & $12 \mathrm{~g} / \mathrm{L}$ \\
\hline Emulsificante & $24 \mathrm{~g} / \mathrm{L}$ \\
\hline $\mathrm{Ca}(\mathrm{OH})_{2}$ & $30 \mathrm{~g} / \mathrm{L}$ \\
\hline Salmuera $\mathrm{CaCl}_{2} 22 \%$ & $0,18 \mathrm{~L}$ \\
\hline $\mathrm{CaCO}_{3}$ & $60 \mathrm{~g} / \mathrm{L}$ \\
\hline Control de filtrado 1 & $10 \mathrm{~g} / \mathrm{L}$ \\
\hline Baritina & $450 \mathrm{~g} / \mathrm{L}$ \\
\hline Control de filtrado 2 & $12 \mathrm{~g} / \mathrm{L}$ \\
\hline
\end{tabular}

La formulación tiene una densidad de $1.300 \mathrm{~kg} / \mathrm{m}^{3}(10.84 \mathrm{lb} / \mathrm{gal})$. La emulsión inversa preparada tiene una proporción volumétrica de 80:20, donde el diesel es la fase continua y la salmuera la discontinua. El diesel es marca Shell y su nombre comercial en Argentina es "Fórmula Diesel". La arcilla organofílica tiene la función de aumentar la viscosidad de la fase continua y dar propiedades tixotrópicas al fluido; el emulsificante facilita y estabiliza la emulsión; el $\mathrm{Ca}(\mathrm{OH})_{2}$ se adiciona para reaccionar con el emulsificante y formar un tensioactivo cálcico; la salmuera de $\mathrm{CaCl}_{2}$ constituye la fase dispersa; el $\mathrm{CaCO}_{3}$ y agentes de filtrado mejoran el control de filtrado, bloquean fracturas y/o fisuras de la roca; la baritina es el densificante del sistema.

La preparación del OBM se hizo siguiendo la secuencia establecida por las normas API 13I (2004) y usando los equipos descriptos en las normas API 13 B2 (2003). Primero se adicionó la arcilla organofilica al diesel y se agitó durante veinte minutos. A continuación, se agregó el emulsificante, luego el $\mathrm{Ca}(\mathrm{OH})_{2}$ en ambos casos se agitó diez minutos. La salmuera se agregó lentamente, se agitó durante treinta minutos y finalmente se agregan los aditivos de control de filtrado y densificante agitando veinte minutos.

Todas las muestras preparadas (WBMs y OBM) fueron envejecidas en un horno de rolado Ofite, modelo 174-00-1, con 3 cilindros rodantes a $25 \mathrm{rpm}$, siguiendo las normas API 13I durante 16 horas a $90^{\circ} \mathrm{C}$, con el propósito de solubilizar los aditivos y simular la circulación del lodo en la sarta de perforación donde existe una degradación mecánica y térmica de algunos aditivos.

Las propiedades reométricas de los fluidos se determinaron en un viscosímetro OFITE modelo 900, con geometría de cilindros coaxiales tipo Couette, usando la configuración R1B1, en un rango de variación de velocidad de corte de $15-850 \mathrm{rpm}$ equivalente a $25,5-1445 \mathrm{~s}^{-1}$, a una temperatura de $25^{\circ} \mathrm{C}$ y en un intervalo de medición constante de $30 \mathrm{~s}$ entre lecturas. Se realizaron dos mediciones para cada velocidad de corte y se calculó el promedio de cada una de las lecturas. Las mediciones que permitieron la construcción de las curvas reológicas, se realizaron a las siguientes velocidades de corte: 25.5, 34, 42.5, 51, 68, 102, 136, 170, 340, 510, $595,680,765,850,935,1020,1105,1190,1275,1360$ y $1445 \mathrm{~s}^{-1}$. La viscosidad plástica y punto de fluencia fueron calculados de acuerdo a lo establecido en las normas API 13B (2003).

Los ensayos de filtrado se realizaron siguiendo las normas API 13B1. Se utilizó un filtro prensa Ofite de baja presión (100 psi), $\mathrm{CO}_{2}$ como gas de presurización a 100 psi y papel de filtro Whatman N50. Las lec- 
turas del filtrado se realizaron a diferentes tiempos: $0.17,5,7.5,10,15,20,25$ y 30 minutos a temperatura ambiente. La primera lectura corresponde al filtrado instantáneo. Por otra parte, se realizaron filtrados de alta presión (500 psi de presión diferencial) y alta temperatura $\left(150^{\circ} \mathrm{C}\right)$ para evaluar la influencia de ambos parámetros. Los ensayos fueron realizados por duplicado y se reportaron los promedios.

Se determinó la concentración de cloruros siguiendo el método de Mohr y la dureza de los fluidos base agua siguiendo las normas API 13 B1.

La densidad de los fluidos se determinó con una balanza presurizada OFITE con un rango de medición de 0,83 a 2,63 gravedad específica. La conductividad eléctrica del OBM fue determinada con un medidor de estabilidad de emulsión OFI en ambos casos se siguieron los procedimientos de las normas API 13B1 y B2.

Los ensayos de dispersión de arcillas, que permiten evaluar el rendimiento del inhibidor, se realizaron siguiendo las normas API 13I. Se prepararon dos fluidos como testigos: agua y solución saturada de $\mathrm{NaCl}$ para ser comparados con $2 \mathrm{WBM}^{*}$ (con y sin inhibidor). Se adicionaron $20 \mathrm{~g}$ de bentonita granulada de 2.000 micrones esperando recuperar su totalidad al finalizar el ensayo. Los WBM* ensayados carecen de baritina y sólidos de formación, dado que estos aditivos no intervienen es esta prueba.

El planteo metodológico de este trabajo de investigación se desarrolló en dos etapas. En la primera, se prepararon los fluidos de perforación WBM y OBM, y se evaluaron sus comportamientos reológicos junto con sus propiedades químicas. En la segunda etapa se adicionaron diferentes concentraciones (\% peso en peso) de nanosílice al WBM: 0,1; 0,3; 0,5 y 1 para determinar la concentración del nanomaterial que permita obtener un WBM con un comportamiento reológico similar al del OBM.

\section{RESULTADOS}

La tabla 3 muestra las propiedades del WBM (sin nanopartículas) y OBM formulados luego de ser envejecidos.

Tabla 3: Propiedades de los fluidos de perforación según ensayos de Norma API 13B1 y 13B2 donde VP es viscosidad plástica y YP es el punto de fluencia.

\begin{tabular}{l|c|c}
\hline \multicolumn{1}{c|}{ PROPIEDAD } & OBM & WBM \\
\hline $\mathrm{VP}(\mathrm{cP})$ a $25^{\circ} \mathrm{C}$ & 54 & 48 \\
\hline $\mathrm{YP}(\mathrm{Pa})$ a $25^{\circ} \mathrm{C}$ & 50 & 51 \\
\hline $\mathrm{Cl}^{-}(\mathrm{mg} / \mathrm{L})$ & 138000 & 4800 \\
\hline Filtrado API (ml) & 0 & 5,2 \\
\hline Filtrado HPHT $(\mathrm{ml})$ & 3 & 164 \\
\hline EE (voltios) & $1068 / 1074$ & 0 \\
\hline
\end{tabular}

En la figura 1 se pueden observar los resultados de los ensayos de dispersión de arcillas realizada a los fluidos testigos y a los WBMs sin envejecer. El WBM* corresponde al fluido sin baritina y sólidos de perforación. 


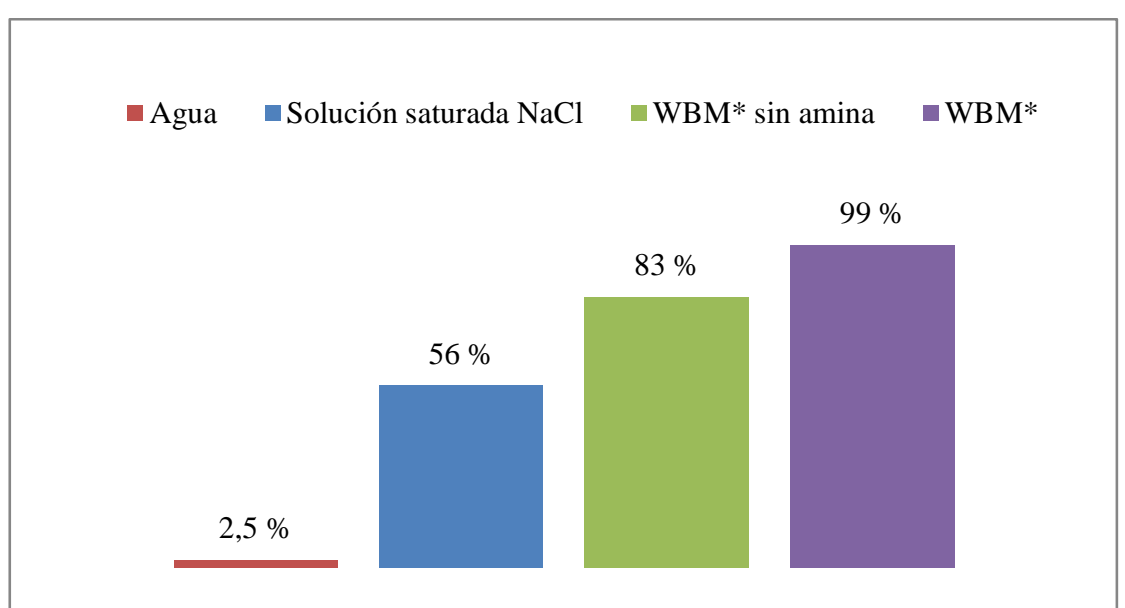

Figura 1: Porcentaje de arcilla recuperada luego de la prueba de inhibición de distintos WBM según las prueba establecida en API Recommended Practice for Laboratory Testing Drilling Fluids 13I (2004).

Las figuras 2 y 3 muestran el comportamiento reológico a $25^{\circ} \mathrm{C}$ del OBM y WBM (sin nanopartículas) antes y después de ser envejecidos. El OBM sin envejecer tiene una conductividad eléctrica entre 763, 771 y 717 voltios.

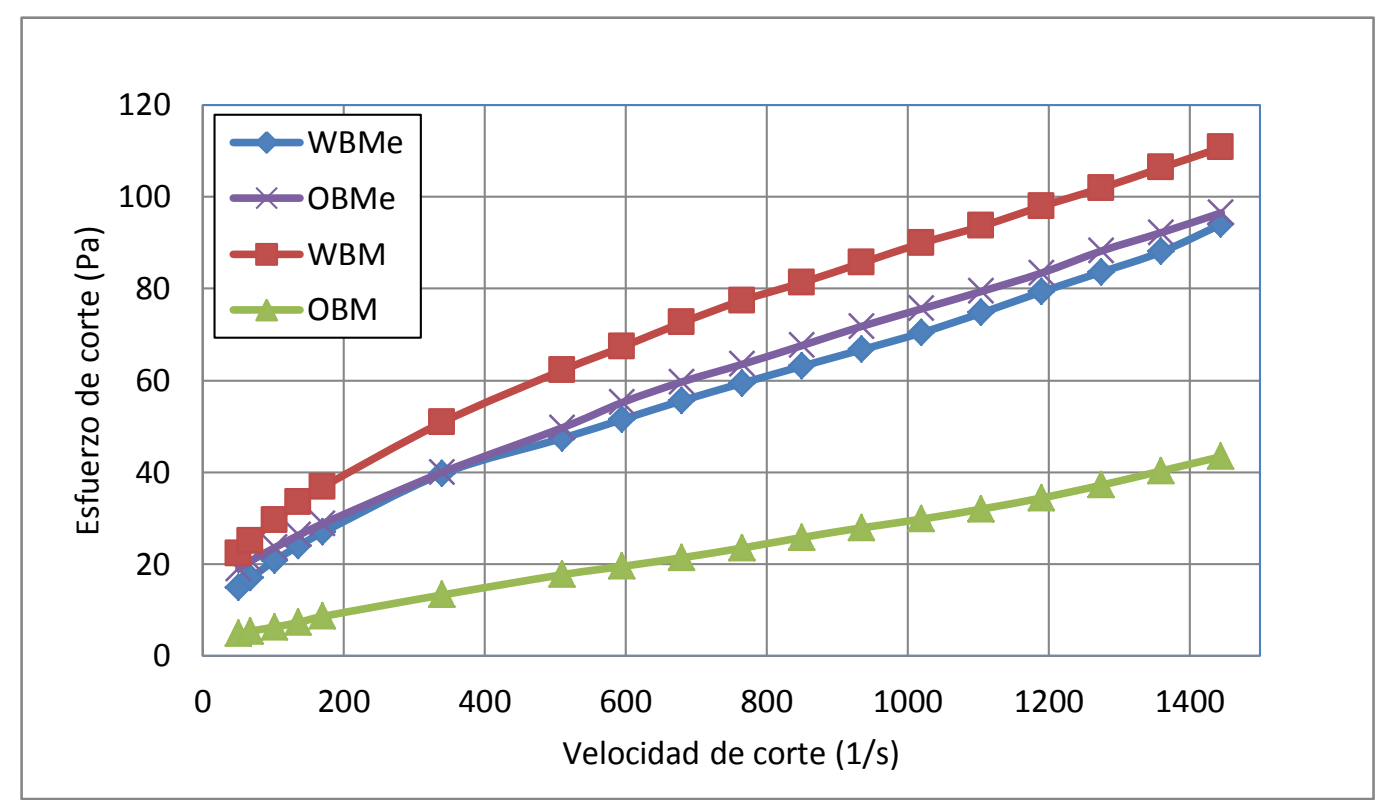

Figura 2: Comportamiento reológico de fluidos de perforación a $25^{\circ} \mathrm{C}$, los fluidos WBMe y OBMe fueron envejecidos en el horno de rolado a $90^{\circ} \mathrm{C}$ durante $16 \mathrm{hrs}$. 


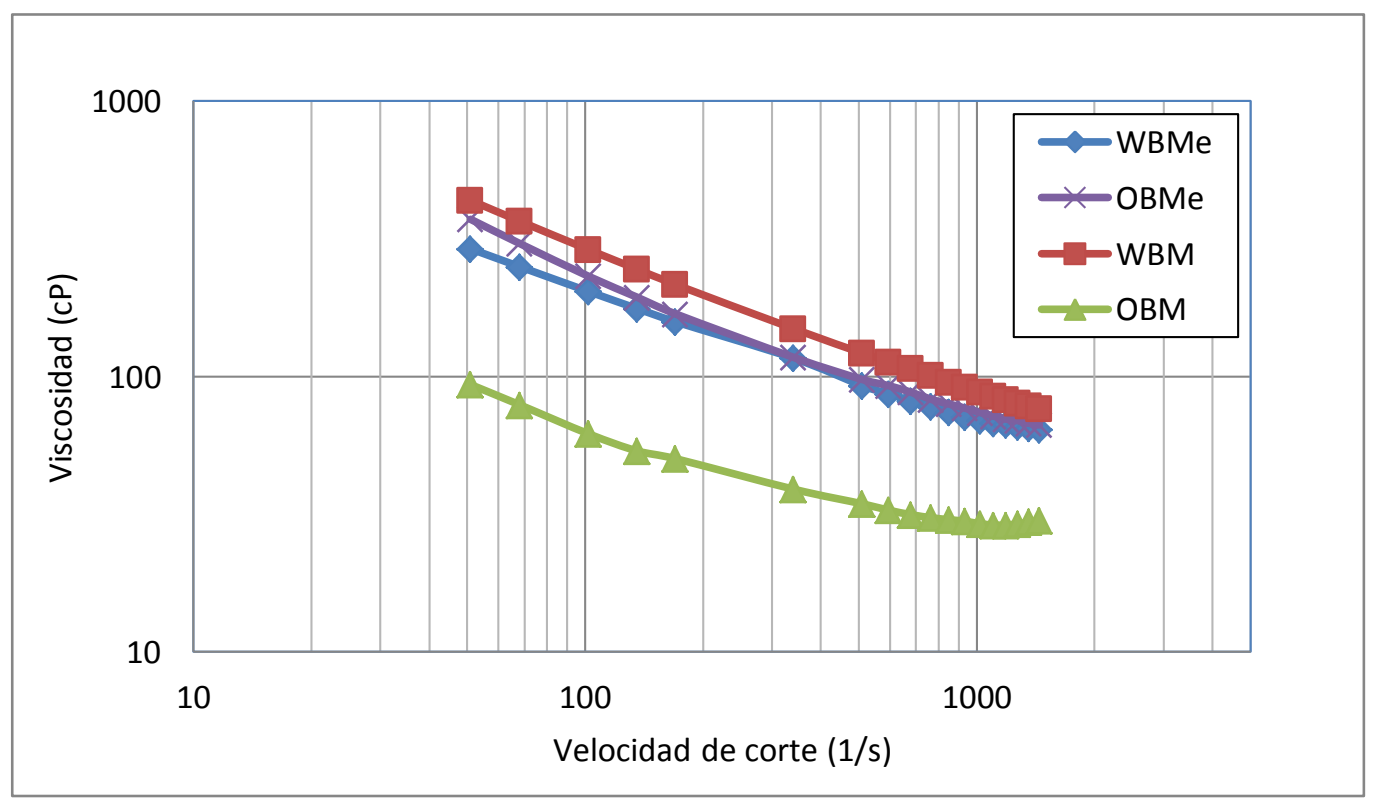

Figura 3: Variación de la viscosidad de fluidos de perforación respeto a la velocidad de corte a $25^{\circ} \mathrm{C}$, $\operatorname{los}$ fluidos $\mathrm{WBMe}$ y OBMe son los envejecidos en el horno de rolado a $90^{\circ} \mathrm{C}$ durante $16 \mathrm{hrs}$

Las figuras 4 y 5 muestran la adición de distintas concentraciones de nanosílice al WBM y su comportamiento reológico a $25^{\circ} \mathrm{C}$. Todos los fluidos fueron envejecidos.

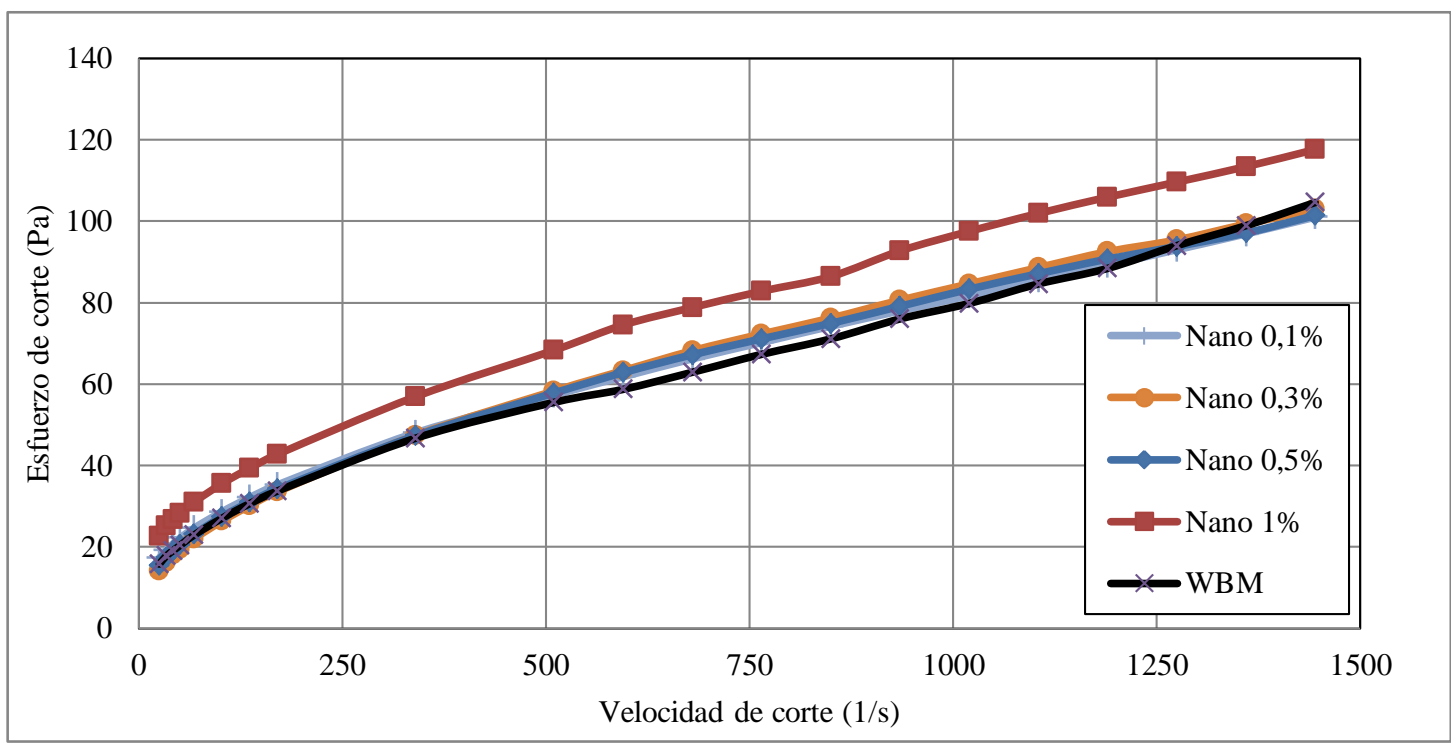

Figura 4: Comportamiento reológico de WBMs a $25^{\circ} \mathrm{C}$ envejecidos en horno de rolado (a $90^{\circ} \mathrm{C}$ durante 16 hrs) variando la concentración de nanosílice. 


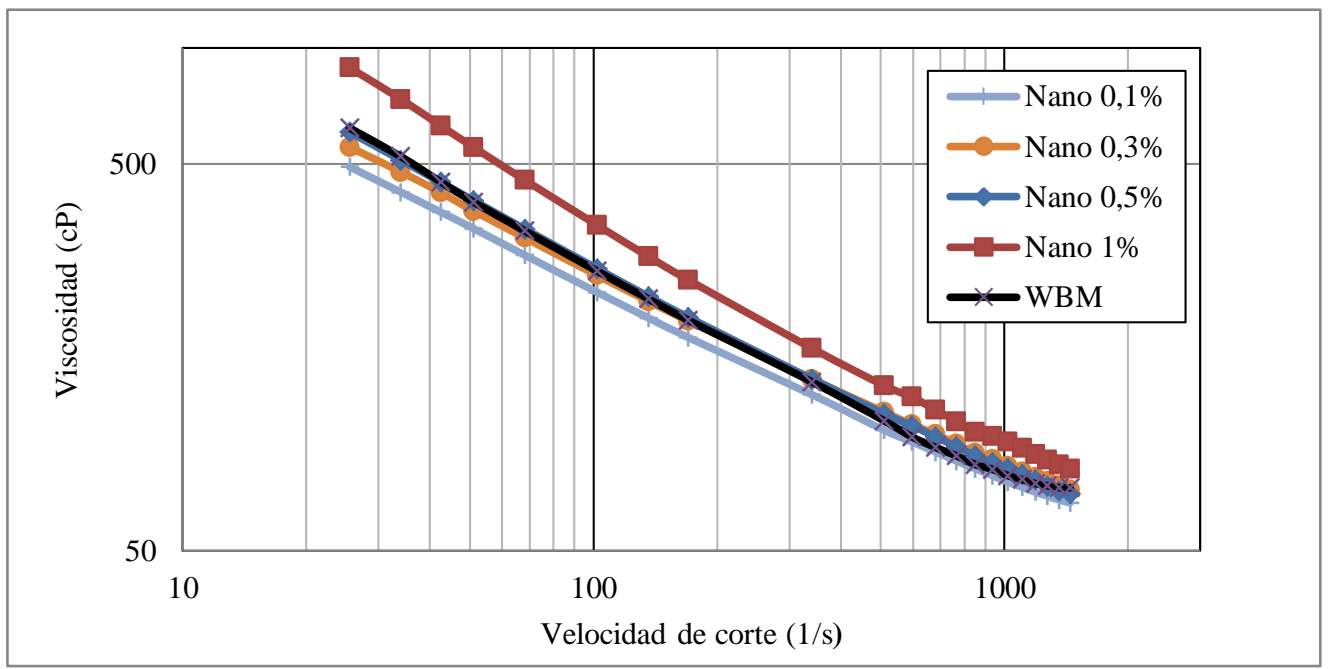

Figura 5: Efecto del agregado de nanosílice \%p/p en la viscosidad de WBMs a $25^{\circ} \mathrm{C}$. Fluidos envejecidos en el horno de roldo a $90^{\circ} \mathrm{C}$ durante 16 hrs.

La tabla 4 muestra los valores viscosidad plástica, punto de fluencia y filtrado API de los fluidos envejecidos.

Tabla 4: Propiedades de WBMs con distintas concentraciones de nanopartículas envejecidos según ensayos de Norma API 13B1. La viscosidad plástica (VP) y punto de fluencia (YP) fueron determinados a $25^{\circ} \mathrm{C}$.

\begin{tabular}{l|c|c|c}
\hline \multicolumn{1}{c|}{ LODO } & VP (CP) & YP (PA) & FILTRADO API (ML) \\
\hline WBM & 51 & 31 & 5 \\
\hline Nano 0.1\% & 41 & 33 & 10 \\
\hline Nano 0.3\% & 48 & 53 & 10 \\
\hline Nano 0.5\% & 46 & 53 & 11 \\
\hline Nano 1\% & 56 & 59 & 14 \\
\hline
\end{tabular}

En la figura 6 se presenta la prueba de filtrado API de los lodos envejecidos.

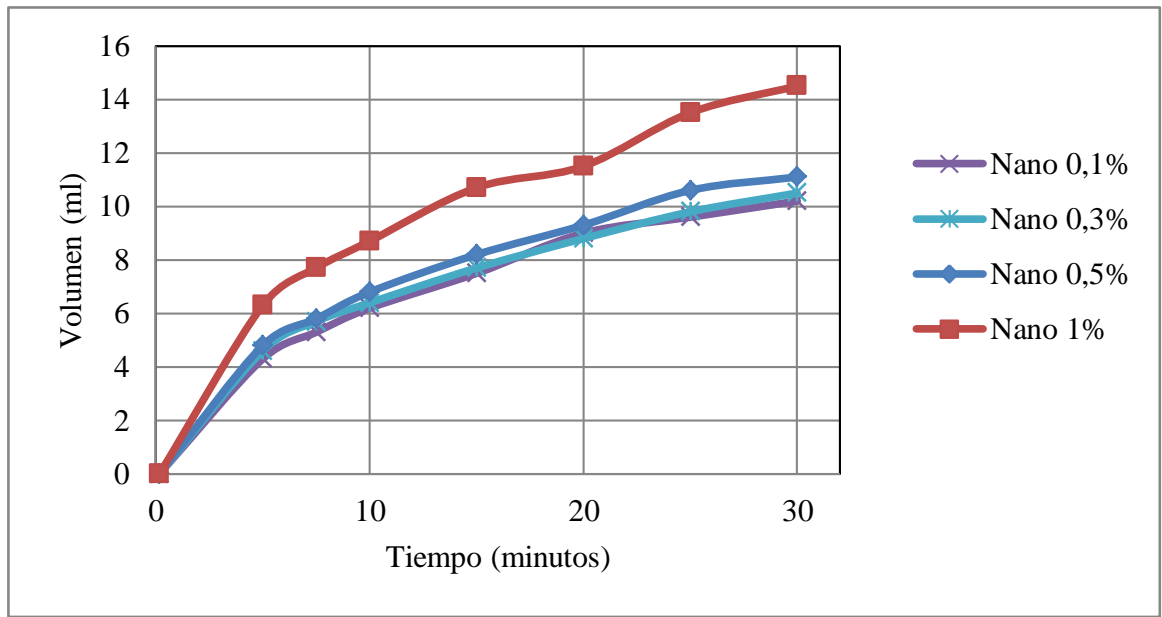

Figura 6: Prueba de filtrado API de WBMs envejecidos en horno de rolado a $90^{\circ} \mathrm{C}$ durante $16 \mathrm{hrs}$. 


\section{DISCUSIÓN}

Los requisitos ambientales cada vez más exigentes han llevado al desarrollo y mejora de los WBMs introduciendo nuevas formulaciones y materiales tales como los nanométricos. En las perforaciones con WBMs se recomienda controlar la presión de hinchamiento y presión poral para alcanzar la estabilidad de las paredes del pozo en el tiempo [2]. En tal sentido los nanomateriales poseen un tamaño adecuado, capaz de bloquear los poros del shale y reducir la presión poral. Además por su gran área superficial, estos nanomateriales, tienen un gran impacto en propiedades como la reología, la cual tiene gran relevancia en estos fluidos por estar relacionada con la limpieza del pozo en el trasporte de los recortes generados por el trépano hacia la superficie.

Los fluidos de perforación estudiados tienen una formulación y aditivos empleados en perforaciones de shale argentino (ver tablas 1 y 2). Estas formulaciones también se condicen con las empleadas en otras regiones del mundo para perforar formaciones con estas características geológicas [8] [19] [20] [21]. En la tabla 3 se muestran las principales propiedades de los fluidos envejecidos, donde la viscosidad plástica y punto de fluencia son próximos entre sí. Esto se debe a que en trabajos previos [22] formularon WBMs variando la concentración del polímero de control de viscosidad y de control de filtrado, para obtener un fluido con características reologicas similares a un OBM. La prueba de dispersión de arcillas (figura1) dio como resultado una alta inhibición de los WBMs* con y sin amina, esto se debe principalmente al agregado del inhibidor y polímero encapsulante. Si bien el $\mathrm{NaCl}$ puede ser considerado un inhibidor, su eficiencia es limitada. La figura 2 muestra la incidencia del ensayo de envejecimiento en los fluidos, donde la temperatura de ensayo seleccionada $\left(90^{\circ} \mathrm{C}\right)$ fue próxima a la temperatura del reservorio. Se estima que durante el envejecimiento la degradación térmica y mecánica de algunos aditivos en el WBM dieron como resultados menores rendimientos. Por el contrario en el OBM la emulsión se estabilizó eléctricamente y la arcilla organofílica (principal aditivo para controlar la viscosidad del OBM) aumentó su rendimiento debido al tratamiento térmico al que fue sometido [23].

El comportamiento reológico de los fluidos fue no newtoniano de acuerdo a las figuras 2 y 3 , el cual puede ser descripto por los modelos reológicos existentes. De acuerdo a diversas investigaciones [24] los fluidos de perforación siguen el modelo de Herschel-Bulkley; son pseudoplásticos y tienen punto de fluencia.

En la tabla 4 su puede observar que la evolución de la viscosidad plástica (cP) en función del agregado de nanosílice no se incrementa en todos los casos con el aumento de nanosílice. Este resultado podría deberse a que la viscosidad plástica es una simplificación que adopta la industria petrolera, al considerar un rango acotado de velocidad de corte entre $511 \mathrm{~s}^{-1}$ a $1022 \mathrm{~s}^{-1}$ asumiendo al fluido como un plástico de Bingham. En la figura 5 se evidencia que la concentración de nanosílice es proporcional al aumento de la viscosidad aparente.

El agregado de $0,5 \%$ p/p de nanosílice fue la máxima concentración a la cual el WBM tuvo un comportamiento reológico similar al OBM a $25^{\circ} \mathrm{C}$, esto se puede observar en las figuras 4 y 5 . En este sentido las concentraciones inferiores mostraron el comportamiento esperado, similar al del WBM. Concentraciones superiores elevaron la viscosidad del fluido hasta valores por encima del rango de medición del viscosímetro y a su vez dificultaron el mezclado del resto de los aditivos por lo cual fueron descartadas. El incremento de la viscosidad puede deberse a la gran área superficial específica de las nanopartículas, $201 \mathrm{~m}^{2} / \mathrm{g}$, que debe ser hidratada por el fluido. La concentración de $0,5 \% \mathrm{p} / \mathrm{p}$ de nanomaterial se encuentra dentro del orden de magnitud de investigaciones previas como la de JIHUA, et al. (2011) [11] y Young, et al. (2013) [12] que sugirió una concentración de 3\% p/p de nanosílice como la óptima si se usan adecuados aditivos de control de filtrado y lubricantes

En futuras investigaciones se analizará el comportamiento reológico del WBM con $0.5 \% \mathrm{p} / \mathrm{p}$ de nanosílice a $65^{\circ} \mathrm{C}$ que corresponde a la temperatura promedio del fluido de perforación luego de circular en el pozo a través de la sarta de perforación y los equipos de superficie. También se trabajará con diferentes presentaciones del nanomaterial (soluciones) y se realizarán ensayos de permeabilidad en medios porosos sintéticos y/o muestras de shale argentino.

\section{CONCLUSIONES}

En este trabajo se estudió un WBM con alta inhibición de arcillas, por la presencia de un polímero encapsulador y amina cuaternaria. Además se agregaron nanopartículas para bloquear las gargantas porales del shale con el objetivo de reducir la presión poral. La goma xantana y celulosa polianiónica contribuyeron a que el filtrado del lodo concuerde con valores aceptables. Con estos aditivos se buscó mejorar la estabilidad de las paredes del pozo en el tiempo. Dado que la reología de los fluidos de perforación es una propiedad diseñada y controlada durante la perforación y que el agregado de nanomateriales la puede modificar sustancialmente, 
se eligió esta propiedad para comparar los comportamientos reológicos entre un OBM y los WBMs con nanopartículas. Los resultados determinaron como máxima una concentración de nanosílice de $0,5 \%$ p $/ \mathrm{p}$ sin que se modifique el comportamiento reológico. En función de las propiedades evaluadas se puede concluir que el lodo obtenido es de alto rendimiento, capaz de estabilizar las paredes del pozo y los recortes generados por el trepano, con un comportamiento reológico optimizado.

\section{BIBLIOGRAFÍA}

[1] APALEKE A. S., BDULAZIZ A., HOSSAIN M. E., "Drilling Fluid: State of The Art and Future Trend", In: North Africa Technical Conference and Exhibition, SPE 149555, Cairo Egypt, 20-22 February, 2012.

[2] VAN OORT, "On The Physical and Chemical Stability of Shales", Journal of Petroleum Science and Engineering, v. 38, pp 213-235, 2003.

[3] VAN OORT, E., HALE, A.H., MODY, F.K., "Manipulation of coupled osmotic flows for stabilisation of shales exposed to water-based drilling fluids", In: SPE Annual Technical Conference and Exhibition, SPE 30499, Dallas Texas, 22-25 October, 1995.

[4] VAN OORT, E., HALE, A.H., MODY, F.K., 1996a, "Transport in shales and the design of improved water-based shale drilling fluids", Journal SPE Drilling \& Completion, v. 11, pp137-146, SPE 28309, 1996.

[5] CHANG M. J., HANG R., CHENEVERT M., et al., "High-Performance Water-Based Mud Using Nanoparticles for Shale Reservoirs", In: Unconventional Resources Technology Conference, SPE 168799/URTeC 1581549, Denver Colorado USA, 12-14 August, 2013.

[6] HOELSCHER K. P., STEFANO G., RILEY M., et al., "Application of Nanoparticles in Drilling Fluids", Journal of Petroleum Technology, v. 64, pp 87-90, SPE 157031, 2012.

[7] LI G., ZHANG J., ZHAO H., HOU Y., "Nanotechnology to Improve Sealing Ability of Drilling Fluids for Shales with Micro-Cracks During Drilling", In: SPE International Oilfield Nanotechnology Conference and Exhibition, SPE 156997, Noordwijk The Netherlands, 12-14 June, 2012.

[8] ARVIND D. P., "Design and Development of Quaternary Amine Compounds: Shale Inhibition with Improved Environmental Profile", In: SPE International Symposium on Oilfield Chemistry, SPE 121737, The Woodlands Texas, 20-22 April, 2009.

[9] Abrams A., "Mud Design to Minimize Rock Impairment due to Particle Invasion", Journal of Petroleum Technology, v. 29, pp 586-592, SPE-5713-PA, 1997.

[10] SURI A., SHARMA M. M., "Strategies for Sizing Particles in Drilling and Completation Fluids", SPE Journal, v. 9,pp 13-23, SPE-87676-PA, 2004.

[11] JIHUA C., CHENEVERT M. E., MUKUL M. S., et al.," Decreasing Water Invasion into Atoka Shales Using Non-Modified Silica Nanoparticles", In: SPE Annual Technical Conference and Exhibition, SPE 146979, Denver Colorado USA, 30 October-2 November, 2011.

[12] YOUNG S., FRIEDHEIM J, "Environmentally Friendly Drilling Fluids for Unconventional Shale", In: Offshore Mediterranean Conference and Exhibition, OMC-2013-102, Ravenna Italy, 20-22 March 2013.

[13] AL-BRAZALI T., "Factors Controlling the Membrane Efficiencie of Shales When Interacting with Water-Based and Oil Based Muds", In: International Oil \& Gas Conference and Exhibition in China, SPE 100735-MS, Beijing China, 5-7 December, 2006.

[14] SENSOY T., CHENEVERT M. E., SHARMA M., "Minimizing Water Invasion in Shales Using Nanoparticles", In: SPE Annual Technical Conference and Exhibition, SPE 124429, New Orleans Louisiana, 4-7 October, 2009.

[15] BARREDO S., STINCO L., "Unconventional Reservoir Geology of the Neuquén Basin Argentina", In: SPE Annual Technical Conference and Exhibition, SPE 170905, Amsterdam The Netherlands, 27-29 October, 2014.

[16] FONTOURA S. A. B., RABE C., LOMBA R. F., "Characterization of Shales for Drilling Purposes", In: SPE/ISRM Rock Mechanics Conference, SPE 78218, Irving Texas, 20-23 October, 2002.

[17] GOMEZ S., WENWU H., "Fighting Wellbore Instability: Customizing Drilling Fluids Based on Laboratory Studies of Shale-Fluid Interactions", In: IADC/SPE Asia Pacific Drilling Technology Conference and Exhibition, SPE 155536, Tianjin China, 9-11 July, 2012.

[18] DARLEY H. C. H. and G. R. GRAY, Composition and Properties of Drilling and Completation Fluids, Fifth Edition, Gulf Professional Publishing, 1988. 
[19] GHOLIZADEH-DOOMECHALY N., TAHMASBI K., DAVANI E., "Development of HighPerformance Water-Based Mud Formulation Based on Amine Derivatives", In: SPE International Symposium on Oilfield Chemistry, SPE 121228, The Woodlands Texas USA, 20-22 April, 2009.

[20] CONTRERAS O., HUSEIN M., NYGAARD R., ALSABA M., “Application of In-House Prepared Nanoparticles as Filtration Control Additives to Reduce Formation Damage", In: SPE International Symposium and Exhibition on Formation Damage Control, SPE 168116, Lafayette Louisiana USA, 26-28 February, 2014.

[21] EWY R. T. Y MORTON E. K., "Wellbore Stability Performance of Water Based Mud Additives", Journal SPE Drilling \& Completion, v. 24, pp. 390-387, SPE 116139, 2008.

[22] GALLARDO F., ERDMANN E., ABALOS. R., "Comportamiento reológico de OBM y WBM para shales argentinos". In: 87 Rane Seguridad de Procesos en Exploración y Producción de Petróleo y Gas ARPEL, Bogotá Colombia, 9-10 marzo, 2016.

[23] Manual de Fluidos de Perforación - MI Swaco - Revisión: A-1 (14-02-2001).

[24] SAFI B., ZAROURI S., CHABANE-CHAOUACHE R., et al., "Physico-Chemical and Rheological Characterization of Water-Based Mud in the Presence of Polymers", Journal of Petroleum Exploration and Production Technology, v. 6, pp.185-190, 2016. 